\title{
Electron-phonon scattering in disordered metallic films
}

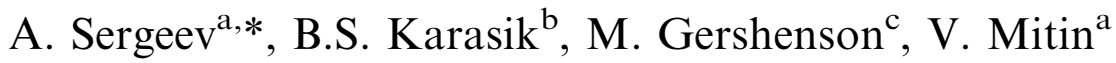 \\ ${ }^{a}$ Department of ECE, Wayne State University, Detroit, MI 48202, USA \\ ${ }^{\mathrm{b}}$ Jet Propulsion Lab., California Institute of Technology, Pasadena, CA 91109, USA \\ ${ }^{\mathrm{c}}$ Department of Physics, Rutgers University, Piscataway, NJ 08854, USA
}

\begin{abstract}
The quantum interference between 'pure' electron-phonon and electron-boundary/impurity scattering drastically changes the electron-phonon relaxation rate. If impurities and boundaries vibrate in the same way as the host lattice, the electron-phonon relaxation rate is significantly decreased. In the presence of the scattering potential that does not vibrate with phonons (e.g. rigid boundaries, interelectron scattering) the relaxation rate is substantially enhanced. Current work reviews recent progress in the theoretical investigations, gives quantitative explanations of available lowtemperature data, and presents original experimental results for ultrathin $\mathrm{Hf}$ at ultralow temperatures. (C) 2002 Elsevier Science B.V. All rights reserved.
\end{abstract}

Keywords: Electron-phonon interaction; Disorder

The electron-phonon relaxation rate in impure materials and thin-film structures has been intensively studied for years. However, current understanding of the electron-phonon interaction (EPI) in disordered conductors is still very limited. The standard clean-limit concept is often applied to disordered conductors, but its uncritical application leads to controversial conclusions. Below we summarize theoretical results (1), give quantitative explanations of electron-heating experiments (2), and presents our experimental results for ultrathin Hf films at ultralow temperatures (3).

(1) EPI is drastically modified in disordered conductors. Diffusive electron motion enhances the 'pure' EPI. Electron scattering from vibrating impurities and boundaries generates another channel of EPI in disordered conductors. Various

*Corresponding author. Tel.: + 1-313-577-5507; fax: + 1313-577-1101.

E-mail address: sergeev@ciao.eng.wayne.edu (A. Sergeev). scattering processes interfere one with another, and the interference results in nontrivial changes of kinetic and transport coefficients.

The investigated model includes vibrating scatterers, which move in same way as host atoms, and additional scatterers, which are not moved by phonons [1]. Assuming short-range random potentials, we introduce the electron mean free path with respect to the nonvibrating potential $(L)$, and the total electron mean free path $(l)$. Theoretical conclusions may be summarized as follows. The energy relaxation rate of electrons interacting with longitudinal phonons is

$$
\begin{aligned}
& \frac{1}{\tau_{\mathrm{e}-1 . \mathrm{ph}}(0)}=\frac{7 \pi \zeta(3)}{2} \frac{\beta_{1} T^{3}}{\left(p_{\mathrm{F}} u_{1}\right)^{2}} F_{1}\left(q_{T} l\right), \\
& F_{1}(z)=\frac{2}{7 \zeta(3)} \int_{0}^{A_{1}} \mathrm{~d} x \Phi_{1}(x z)\left(N_{x}+n_{x}\right) x^{2}, \\
& \Phi_{1}(x)=\frac{2}{\pi}\left(\frac{x \arctan (x)}{x-\arctan (x)}-\frac{3}{x}+\frac{l}{L} \frac{3}{x}\right),
\end{aligned}
$$


where $A_{\mathrm{t}(1)}=\theta_{\mathrm{D}} l / u_{\mathrm{t}(1)} z\left(\theta_{\mathrm{D}}\right.$ is the Debye temperature), $p_{\mathrm{F}}$ is the Fermi momentum, $u_{1(\mathrm{t})}$ is the longitudinal (transverse) sound velocity. The dimensionless coupling constants are given by $\beta_{1}=$ $\beta_{\mathrm{t}}\left(u_{\mathrm{t}} / u_{1}\right)^{2}=\left(2 \varepsilon_{\mathrm{F}} / 3\right)^{2} v /\left(2 \rho u_{1}^{2}\right), v$ is the electron density of states.

The energy relaxation rate of electrons due to transverse phonons is

$\frac{1}{\tau_{\mathrm{e}-\mathrm{t} . \mathrm{ph}}(0)}=\frac{3 \pi^{2} \beta_{\mathrm{t}} T^{2}}{\left(p_{\mathrm{F}} u_{\mathrm{t}}\right)\left(p_{\mathrm{F}} l\right)}\left(1-\frac{l}{L}\right) F_{\mathrm{t}}\left(q_{T} l\right)$

$F_{\mathrm{t}}(z)=\frac{4}{\pi^{2}} \int_{0}^{A_{\mathrm{t}}} \mathrm{d} x \Phi_{\mathrm{t}}(x z)\left(N_{x}+n_{x}^{\mathrm{eq}}\right) x$

$\Phi_{\mathrm{t}}(x)=1+\left(1-\frac{l}{L}\right) \frac{3 x-3\left(x^{2}+1\right) \arctan (x)}{2 x^{3}}$.

In the impure case $\left(T l<u_{1}, u_{\mathrm{t}}\right)$, the electronphonon scattering rate is

$$
\begin{aligned}
\frac{1}{\tau_{\mathrm{e}-\mathrm{ph}}(0)}= & \frac{\pi^{4} T^{4} l}{5 p_{\mathrm{F}}^{2}}\left[\frac{\beta_{1}}{u_{1}^{3}}+\left(1-\frac{l}{L}\right) \frac{3 \beta_{\mathrm{t}}}{2 u_{\mathrm{t}}^{3}}\right] \\
& +\frac{3 \pi^{2} T^{2}}{2 p_{\mathrm{F}}^{2} L}\left[\frac{\beta_{1}}{u_{1}}+\left(1-\frac{l}{L}\right) \frac{2 \beta_{\mathrm{t}}}{u_{\mathrm{t}}}\right] .
\end{aligned}
$$

In the case of vibrating scatterers $(L \rightarrow \infty)$ Eqs. (1)-(7) reproduce results of Refs. [2,3].

Eqs. (1) and (4) determine the relaxation of electrons at the Fermi surface. Cooling rate of electron subsystem, $1 / \tau_{\varepsilon}$, is given by

$\frac{1}{\tau_{\varepsilon}}=\frac{3(n+2) \Gamma(n+2) \zeta(n+2)}{2 \pi^{2}\left(2-2^{1-n}\right) \Gamma(n) \zeta(n)} \frac{1}{\tau_{\mathrm{e}-\mathrm{ph}}(0)}$,

where $n$ is exponent in the temperature dependence of relaxation rate, $1 / \tau_{\mathrm{e}-\mathrm{ph}}(0) \propto T^{n}$.

Vibrations of electron scatterers, such as impurities and defects, depends on the mass of scatterer and local force constants. In fact, the difference in vibrations of impurities (defects) and host atoms is not significant [4]. Therefore, a fraction of nonvibrating potential is small, and the ratio $l / L$ may be evaluated as $10^{-4}-10^{-3}$. Note, that this factor may be larger (up to 0.1) in pure thin-film structures on rigid substrates, such as diamond. In any case, in the region of moderate disorder, $q_{T} l \sim 1$, the effect of the nonvibrating potential on the inelastic relaxation rate is small. In the next section we show that experimental data are well described by the Eqs. (1)-(6) with $l / L \rightarrow 0$. Accord- ing to Eq. (7), in the region of strong disorder $\left(q_{T} l \ll 1\right)$ the effect of nonvibrating potential is crucial, if $q l \cdot q L \ll 1$. In this region the electronphonon relaxation substantially depends on the character of electron scatterers (see last section).

(2) Let us compare theoretical predictions and data obtained from electron-heating experiments with $\mathrm{Cu}$ [5] and AuPd [6] films. The $\mathrm{Cu}$ films with high diffusivity $D=200 \mathrm{~cm}^{2} / \mathrm{s}$ were studied at ultralow temperatures, the electron cooling rate, $1 / \tau_{\varepsilon}$, was found to be $9 \times 10^{7} T^{3} \mathrm{~s}^{-1}$ [5]. The AuPd films with diffusivity $D=7.2 \mathrm{~cm}^{2} / \mathrm{s}$ were investigated at helium temperatures, and measured cooling rate was $5 \times 10^{7} T^{3.7} \mathrm{~s}^{-1}[6]$. Data of Refs. $[5,6]$ correspond to the region of moderate disorder. In Figs. 2 and 3 we present the relaxation rate at the Fermi surface (see Eq. (8)) in these films. Using parameters of $\mathrm{Cu}(\mathrm{AuPd}): v_{\mathrm{F}}=1.6 \times$ $10^{8}\left(1.4 \times 10^{8}\right) \mathrm{cm} / \mathrm{s}, \quad v=1.6 \times 10^{41}\left(9.9 \times 10^{40}\right)$ $\mathrm{J}^{-1} \mathrm{~cm}^{-3}, u_{\mathrm{t}}=2.3 \times 10^{5}\left(2 \times 10^{5}\right) \mathrm{cm} / \mathrm{s}$, and $u_{1}=$ $5 \times 10^{5}\left(5 \times 10^{5}\right) \mathrm{cm} / \mathrm{s}$, we calculate the relaxation rate from Eqs. (1)-(6) with $L \rightarrow \infty$. Figs. 1 and 2 show very good agreement of theoretical calculations with experimental results. Note, that in the region of moderate disorder, transverse phonons strongly dominate in the electron-phonon relaxation. The measured temperature dependencies correspond to the intermediate range, where the relaxation rate changes from $T^{4} l$ at $q_{T} l \ll 1$ to $T^{2} l^{-1}$ at $q_{T} l \gg 1$ (see Eqs. (3)-(6)).

(3) We have experimentally investigated the electron-phonon relaxation rate in the region of

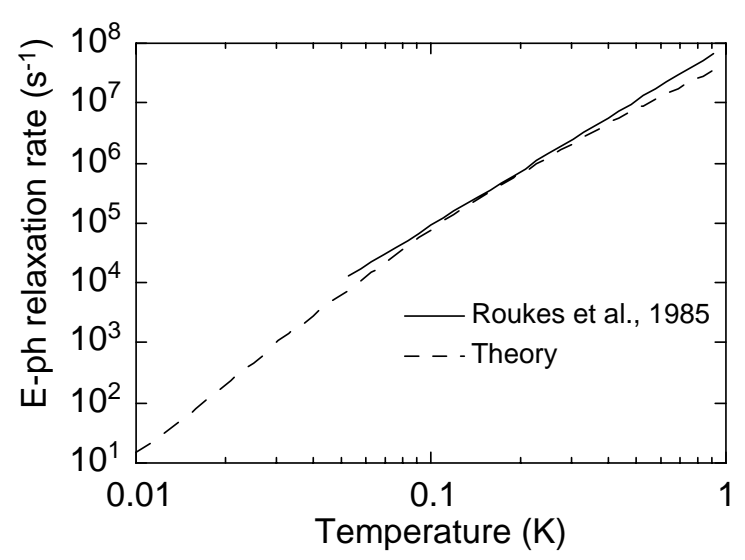

Fig. 1. The electron-phonon relaxation rate in $\mathrm{Cu}$ films [5]. 


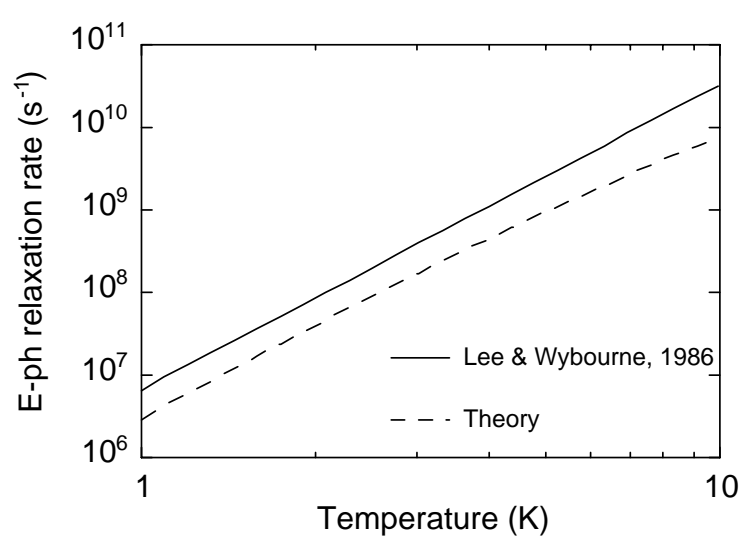

Fig. 2. The electron-phonon relaxation rate in AuPd films [6].

strong disorder. We studied electron cooling in ultrathin Hf films with short electron mean free path $(l=0.94 \mathrm{~nm})$ at millikelvin temperatures $(0.04-1 \mathrm{~K})$. The data obtained from electronheating experiments indicate that the relaxation in these films is significantly suppressed by disorder. Shown in Fig. 3, the electron cooling time follows to $T^{-4}$-dependence with a record-long value of $\tau_{\varepsilon}=25 \mathrm{~ms}$ at $T=0.04 \mathrm{~K}$. Acoustic impedance of Hf is very close to the impedance of sapphire substrate, so vibrations of the filmsubstrate interface are expected to be identical to the phonon modes in the film. Using parameters of Hf: $\quad v_{\mathrm{F}}=0.47 \times 10^{8} \mathrm{~cm} / \mathrm{s}, u_{\mathrm{t}}=1.97 \times 10^{5} \mathrm{~cm} / \mathrm{s}$, we find that the relaxation rate is well described by the theory with $L \rightarrow \infty$.

According to Eq. (7), in the limit of strong disorder the nonvibrating potential may change the temperature dependence of the relaxation rate from $T^{4}$ to $T^{2}$. The dependencies close to $T^{2}$ are widely observed in experiments, but most of the data correspond to the region of moderate disorder, $q_{T} l \geqslant 1$ (contribution of transverse phonons). Recently the $T^{2}$-dependence has been measured in $\mathrm{Ti}_{73} \mathrm{Al}_{27}$ films in the region of strong disorder, $q_{T} l \sim 0.01$ [7]. Relative amplitude of vibrations of $\mathrm{Al}$ atoms with respect to $\mathrm{Ti}$ atoms may be evaluated as $k \sim 1.0001-1.001$ [4], so the

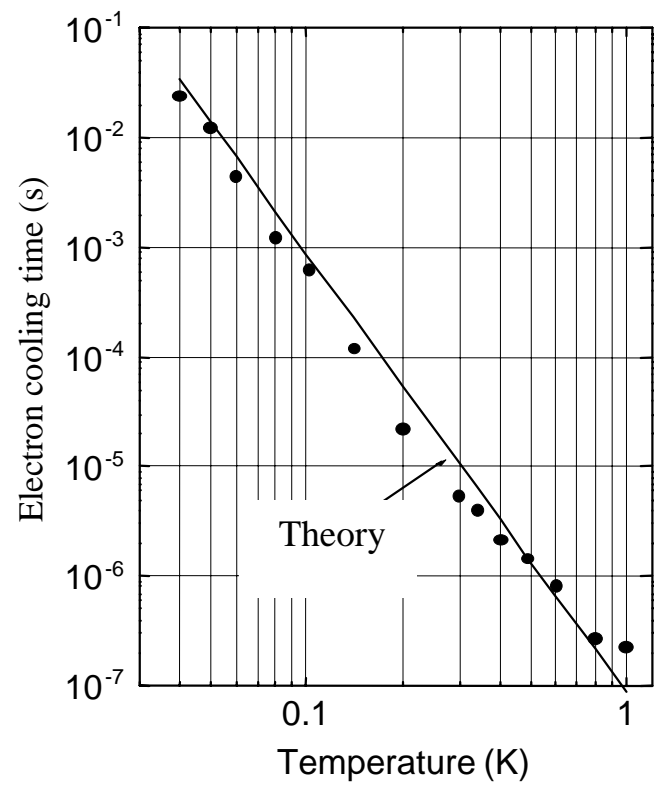

Fig. 3. The electron cooling time in Hf films.

fraction of nonvibrating potential is $l / L \sim$ $(k-1) \simeq 10^{-4}-10^{-3}$, and the parameter $\left(q_{T} l\right)\left(q_{T} L\right)$ is $\sim 0.1-1$. As we already discussed, this parameter describes transition from $T^{4}$ to $T^{2}$ dependence (Eq. (7)). Thus, the observations of Ref. [7] are in qualitative agreement with the theory.

The research was supported by NASA and NSF grants.

\section{References}

[1] A. Sergeev, V. Mitin, Phys. Rev. B 61 (2000) 6041; A. Sergeev, V. Mitin, Europhys. Lett. 51 (2000) 641.

[2] A. Schmid, Z. Phys. 259 (1973) 421.

[3] M.Yu. Reizer, A.V. Sergeev, Zh. Eksp. Teor. Fiz. 90 (1986) 1056 [Sov. Phys. JETP 63 (1986) 616].

[4] H. Bottger, Principles of the Theory of Lattice Dynamics, Physica-Verlag, Berlin, 1983.

[5] M.L. Roukes, et al., Phys. Rev. Lett. 55 (1985) 422.

[6] R.A. Lee, M.N. Wybourne, J. Phys. F 16 (1986) L169.

[7] C.Y. Wu, W.B. Jian, J.J. Lin, Phys. Rev. B 57 (1998) 11232. 